\title{
Practical Service Chaining based on IP Routing
}

\author{
Ryo Nakamura \\ The University of Tokyo \\ Kazuki Shimizu \\ Juniper Networks
}

\author{
Yukito Ueno \\ NTT Communications \\ Takashi Tomine \\ Lepidum Co. Ltd.
}

\author{
Teppei Kamata \\ Cisco Systems \\ Takayuki Watanabe
Juniper Networks
}

\begin{abstract}
In this paper, we demonstrate that practical service chaining can be achieved by leveraging existing and standardized IP routing techniques. Service chaining is an emerging network concept for dynamically applying network services to traffic. A straightforward for service chaining is to utilize software-defined networking techniques such as OpenFlow. However, they require additional development costs on both control and data planes and introduce complexity into operation from the practical viewpoint. Our proposed method, called Flowchain, enables us to construct service chaining with standardized IP routing protocols by exploiting the redirect action of BGP Flowspec. Building service chaining with IP routing reduces development cost on both planes and make network management easy. This paper describes how Flowchain achieves service chaining, and our experiments show the feasibility of Flowchain through a demonstration of service insertion and deletion, evaluation of the time required to insert a new service chain, and failover tests.
\end{abstract}

\section{CCS CONCEPTS}

- Networks $\rightarrow$ Network structure; Network manageability;

\section{KEYWORDS}

Service Chaining; IP Routing; BGP Flowspec.

\section{ACM Reference Format:}

Ryo Nakamura, Yukito Ueno, Teppei Kamata, Kazuki Shimizu, Takashi Tomine, and Takayuki Watanabe. 2018. Practical Service Chaining based on IP Routing. In AINTEC '18: ASIAN INTERNET ENGINEERING CONFERENCE (AINTEC '18), November 12-14, 2018, Bangkok, Thailand. ACM, New York, NY, USA, 8 pages. https://doi.org/10. $1145 / 3289166.3289170$

Permission to make digital or hard copies of all or part of this work for personal or classroom use is granted without fee provided that copies are not made or distributed for profit or commercial advantage and that copies bear this notice and the full citation on the first page. Copyrights for components of this work owned by others than ACM must be honored. Abstracting with credit is permitted. To copy otherwise, or republish, to post on servers or to redistribute to lists, requires prior specific permission and/or a fee. Request permissions from permissions@acm.org.

AINTEC '18, November 12-14, 2018, Bangkok, Thailand

(c) 2018 Association for Computing Machinery.

ACM ISBN 978-1-4503-6131-6/18/11 ...\$15.00

https://doi.org/10.1145/3289166.3289170

\section{INTRODUCTION}

The diversification of network clients, users, and applications requires network infrastructures to apply proper network services to their traffic flexibly. Client devices should be protected by security services, e.g., Firewall and Deep Packet Inspection (DPI), web servers sometimes need Web Application Firewalls (WAF), and operators often apply captive portals to wireless clients by their policy. Service chaining is an emerging network concept to meet this demand. In service chaining, such middleboxes are called service functions, which are responsible for network services, and a set of service functions applied to a series of packets is called a service chain (or a Service Function Chain [8]).

A promising approach for achieving service chaining is to leverage software-defined networking (SDN) $[9,12]$, although SDN requires additional development costs and often introduces complexity into network operation [19]. As an implementation of service chains in networks, service chains become paths where classified packets flow. Each path steers packets through expected service functions in a configured order. SDN technologies, especially OpenFlow, can implement such path-based packet forwarding mechanisms for service chaining as shown in previous studies $[4,13,15,21]$. However, when considering the realistic deployment and operation of service chaining with OpenFlow, network operators need to develop controller software specialized in their control logic. Moreover, network operators must be well versed in their dedicated packet forwarding mechanisms to debug network faults, although managing and debugging SDN are still a developing research area [18].

In contrast, traditional IP routing, which is well matured and in which many operators are skilled, lacks the functionality to implement the paths in networks because it is hopby-hop, shortest-path routing. In IP routing, a destination always has one next-hop (or equal-cost next-hops), and there are no alternative or arbitrary paths. Therefore, industrial and research communities tend to adopt other packet forwarding mechanisms to achieve the paths in networks, such as Network Service Header (NSH) [16], Multi-Protocol Label Switching (MPLS) [5], Segment Routing [6], and OpenFlow, although these may add additional complexity to network deployment and management. 
Table 1: Comparison between SDN and traditional IP routing from the viewpoint of realistic deployment and operation of service chaining.

\begin{tabular}{c|c|c|c}
\hline & Functionality & Development cost & Management \\
\hline SDN & $\checkmark$ & High & Difficult \\
IP & & Low & Easy \\
\hline
\end{tabular}

SDN and new packet forwarding mechanisms will undoubtedly achieve service chaining practically. Before they have matured, we revisit IP routing in this paper. Table 1 shows a comparison between SDN and IP for realistic service chaining deployment. If service chaining is achieved with IP routing, it offers low development cost and easy management based on well-matured, commercial off-the-shelf IP routers and the knowledge that the network communities have accumulated. However, IP routing cannot achieve path-based packet forwarding to implement service chains.

To fill the gap in IP routing shown in Table 1, we propose to exploit BGP Flow Specification (Flowspec) [11]. BGP Flowspec was initially designed to distribute access control list information over BGP for filtering denial-of-service attacks. Our proposed method, called Flowchain, diverts this BGP Flowspec to service chaining. The key idea is to utilize redirection instead of paths implemented in networks. We achieve service chains by redirecting targeted traffic from/to individual IP networks accommodating service functions using the redirect action of BGP Flowspec. Flowchain uses only standardized protocols, so that its management is easy compared with SDN-based dedicated mechanisms because its data plane and control plane are familiar IP routing. Therefore, we argue that Flowchain is a practically deployable and operable service chaining method at present.

To demonstrate Flowchain, we implemented a simple BGP Flowspec speaker, which is just about 1000 lines of codes, using ExaBGP [3], and constructed a simple testbed with two Juniper Networks MX5 routers. Our experiments on the testbed show the feasibility of Flowchain through a demonstration of service insertion and deletion. Moreover, other experiments show that Flowchain can change service chains dynamically within a few seconds, and practical failover using Open Shortest Path First (OSPF).

The contributions of this paper include the following:

- We demonstrate that service chaining can be achieved with IP routing techniques: BGP Flowspec and OSPF.

- The experiments demonstrate the potential feasibility of achieving practical service chaining.

We believe that IP-based service chaining would contribute to future comparison and discussion of service chaining methods based on SDN and new packet forwarding mechanisms.

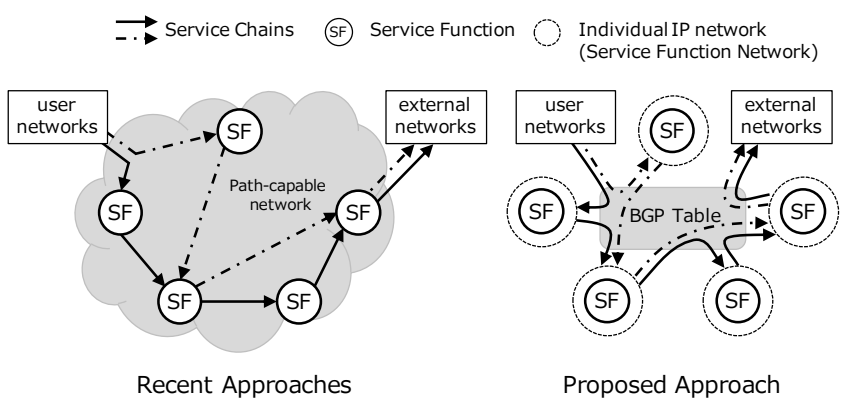

Figure 1: Recent approaches require networks and/or service functions to be capable of handling the paths. In contrast, the proposed approach achieves the service chains with redirection by BGP Flowspec routes.

\section{APPROACH}

To achieve service chaining with IP routing, we divert BGP Flowspec to steer packets instead of non-IP-based packet forwarding that implements paths in networks. Figure 1 illustrates the differences between recent and proposed approaches. In the recent approaches, network components need to be aware of paths to handle packets. The network components might be software switches in Hyper Visors like OpenvSwitch, or hardware devices like OpenFlow switches. In either case, controllers program the network components and/or service functions to steer packets along the paths.

In contrast, we utilize redirection to achieve service chains. The redirect extended community of BGP Flowspec enables us to steer packets from/to service functions at BGP routing tables. BGP Flowspec brings the access control list information as Network Layer Reachability Information (NLRI) that contains match rules (destination and source addresses, port numbers, etc.) and actions (drop, marking, etc.). Routers that receive Flowspec routes install the routes into their packet forwarding engines, and process packets following the routes. The redirect extended community is an action of BGP Flowspec. It redirects matched packets to target Virtual Routing and Forwarding (VRF) instances, which are individual routing tables on a single router.

Figure 1 also shows how to achieve service chains with the redirection of BGP Flowspec. Each service function is accommodated in an individual IP network built by VRF instances (a service function network). The Flowspec routes installed in the routing table redirect packets from the service function networks to other service function networks. As a result, the packets pass through the proper service functions in specific orders, which are the service chains, without pathcapable packet forwarding mechanisms.

One advantage of this approach is to use commercial offthe-shelf IP routers without data plane programming. BGP 
Practical Service Chaining based on IP Routing

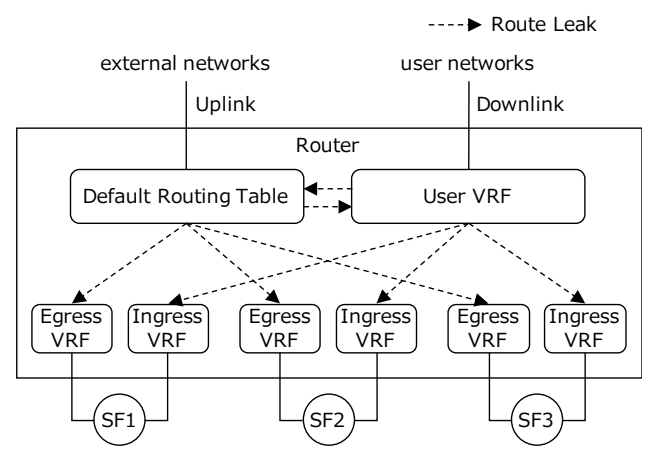

Figure 2: The basic setup of VRFs in a router accommodating service functions.

Flowspec was initially designed to filter denial-of-service attacks, and was widely implemented in various router products [10]. Thus, we can divert existing BGP Flowspec-capable routers and their packet forwarding engines as is. There is no development cost to implement dedicated controllers and data planes. Another advantage is to use IP networks to accommodate service functions. The service functions just forward packets in accordance with traditional IP routing; therefore, we can use existing middleboxes as service functions. Moreover, matured methods for redundancy in IP networks can also be applied to the service function networks, for example, OSPF and Bidirectional Forwarding Detection.

\subsection{Flowchain}

To construct practical service chaining, we propose Flowchain as the detailed network structure based on the key idea of exploiting the VRF redirect of BGP Flowspec. Flowchain is composed of two steps: the basic routing table setup and installing Flowspec routes.

2.1.1 Basic Setup. At the first step, routers accommodate each service function in a network using VRF instances and make each service function network have the default route and user prefixes as destinations. Figure 2 illustrates a router that accommodates three service functions. The service functions connect to the pairs of two VRFs: ingress VRF and egress VRF. Individual OSPF processes run on each VRF so that the ingress and egress VRFs exchange routes across the service function. If the service function is a layer-2 inline middlebox, the associating ingress and egress VRFs establish the OSPF neighbor relationship directly. If the service function is a layer-3 middlebox, the VRFs and the middlebox exchange the routes using OSPF. Two other routing tables, the default routing table and the user VRF, connect to the uplink and the downlink, respectively. Both routing tables also run individual OSPF processes and exchange routes with external networks and user networks.
AINTEC '18, November 12-14, 2018, Bangkok, Thailand

Table 2: The routing table entries in Figure 2.

\begin{tabular}{c|c|c}
\hline VRF & Destination & Next-hop \\
\hline $\begin{array}{c}\text { Default } \\
\text { Routing Table }\end{array}$ & $\begin{array}{c}0.0 .0 .0 / 0 \\
\text { user prefixes }\end{array}$ & $\begin{array}{c}\text { Uplink } \\
\text { Downlink }\end{array}$ \\
\hline User VRF & $\begin{array}{c}0.0 .0 .0 / 0 \\
\text { user prefixes }\end{array}$ & $\begin{array}{c}\text { Uplink } \\
\text { Downlink }\end{array}$ \\
\hline Egress VRF & $\begin{array}{c}0.0 .0 .0 / 0 \\
\text { user prefixes }\end{array}$ & $\begin{array}{c}\text { Uplink } \\
\text { Ingress VRF or SF }\end{array}$ \\
\hline Ingress VRF & $\begin{array}{c}0.0 .0 .0 / 0 \\
\text { user prefixes }\end{array}$ & $\begin{array}{c}\text { Egress VRF or SF } \\
\text { Downlink }\end{array}$ \\
\hline $\begin{array}{c}\text { Service Function } \\
\text { (if it is layer-3) }\end{array}$ & $\begin{array}{c}0.0 .0 .0 / 0 \\
\text { user prefixes }\end{array}$ & $\begin{array}{c}\text { Egress VRF } \\
\text { Ingress VRF }\end{array}$ \\
\hline
\end{tabular}

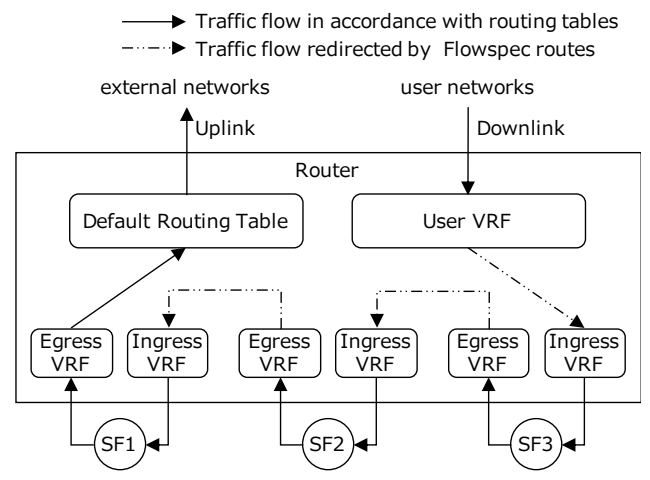

Figure 3: An example of installing Flowspec routes for a service chain. Packets from the user network pass through SF3, SF2, and SF1 by redirection.

In addition, we populate routing table entries between the VRFs to forward packets up and down. Importing or exporting routes from a VRF to other VRFs, which is also known as route leaking, is a popular functionality implemented in BGP/VPN-capable routers [17]. As shown in Figure 2, the default routing table and the user VRF leak all routes to each other. Moreover, the default routing table leaks the default route to all egress VRFs, and the user VRF leaks all the user prefixes to all ingress VRFs. Table 2 shows routing table entries as a result of this route leaking. The router forwards packets between the uplink and the downlink, and each service function network forwards packets up and down in accordance with the destinations of the packets.

2.1.2 Installing Flowspec Routes. After basic routing table setup, Flowspec routes can construct service chains. Figure 3 shows an example of a service chain passing through SF3, SF2, and SF1. For the traffic from a target user network to destinations, the Flowspec routes match the source addresses of the packets. If the source addresses match the target user 
prefix, the matched packets are redirected to the ingress VRF of the next service function. The first Flowspec route is installed in the user VRF. It matches the source addresses and redirects the packets to the ingress VRF of SF3. The ingress VRF transmits the packets to SF3, and the egress VRF of SF3 receives the packets. The next Flowspec route is installed in this egress VRF. This route also matches the source addresses and then redirects the packets to the ingress VRF of the next service function, SF2. Finally, the egress VRF of SF1 transmits packets to the uplink in accordance with the default route leaked from the default routing table. Flowchain achieves service chaining with BGP Flowspec in this manner.

For the packets from external to the user network, reversed Flowspec routes steer packets in the reverse order. The reversed Flowspec routes match the destination addresses. The first route is installed in the default routing table. If destination addresses of the packets match the target user prefix, the route redirects the packets to the egress VRF of SF1. After the ingress VRF of SF1 receives the packets through SF1, the next Flowspec routes also match the destination addresses, and redirect the packets to the egress VRF of SF2. By repeating this process, ingress traffic and egress traffic associating the user prefix pass through the symmetric service chain.

\subsection{Integrating Multiple Routers}

The previous section described how to construct service chains with one router. As expected, building service chaining with a single router lacks expandability. In this section, we describe how to build Flowchain-based service chaining with multiple routers.

To construct service chains across multiple routers, we need to bring all packets having arbitrary destinations between the routers obeying IP routing. To do this, we emulate unidirectional point-to-point links between the routers. Figure 4 depicts two routers forming service chaining. Both routers completed the basic setup described in Section 2.1.1. In addition, they have two VRFs to transmit and receive packets to and from the opposite router. To-R2 VRF on Router 1 and From-R1 VRF on Router 2 are connected via a link (e.g., a link, a VLAN, an MPLS path, or a tunnel), and they establish the OSPF neighbor relationship across the inter-router link. Moreover, From-R1 VRF originates the default route to To-R2 VRF. As a result, To-R2 VRF on Router 1 sends all packets redirected to To-R2 VRF to From-R1 VRF on Router 2.

Next, From-R1 VRF on Router 2 needs to distribute packets to proper VRFs; however, we cannot use address matching of Flowspec. When a service chain crosses the inter-router link multiple times, address matching on From-R1 VRF cannot forward packets to different VRFs for a second time. Therefore, we utilize the Type-of-Service (ToS) value of the IP header. Flowspec routes on From-R1 VRF redirect packets

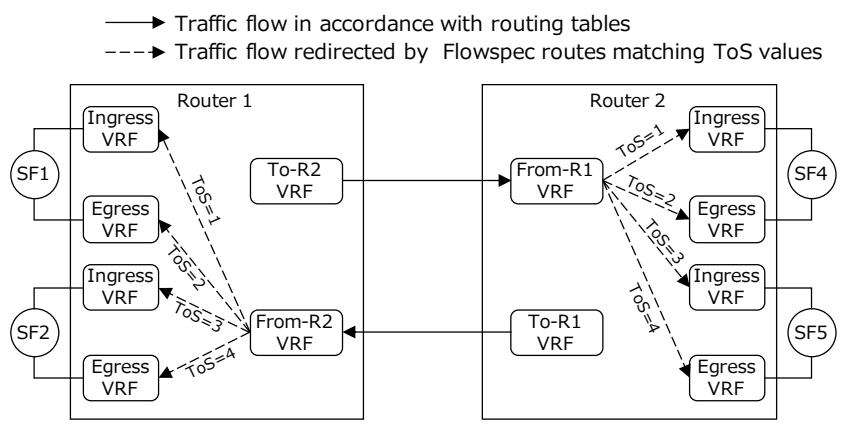

Figure 4: Constructing service chaining with two routers. Two unidirectional point-to-point links bring packets to each router.

to VRFs matching the ToS value. When a Flowspec route redirects packets to To-R2 VRF on Router 1, this route also marks the packets with a ToS value corresponding to the next service function. As a result, the Flowspec route on From-R1 VRF can redirect the packets to the proper VRF using the ToS value of the packets. The same applies to the case from Router 2 to Router 1 . In addition, full mesh pointto-point links can incorporate over two routers into a single service chaining infrastructure. By this method, Flowchain integrates multiple routers into a single service chaining infrastructure.

Note that ToS matching of BGP Flowspec uses 6-bit Diffserv Code Point [1]. Thus, the number of service functions on a single router is limited to 32 because Flowchain uses two VRFs-ingress and egress-for each service function.

\subsection{Failover}

Fault tolerance is an essential part for practical deployment of service chaining. In Flowchain, Failover, which is an important functionality for fault tolerance, is classified into two types: service function-level failover and router-level failover. Both failures can recover relying on OSPF.

Service Function-level Failover: Service function networks are IP networks; therefore, we can adopt traditional failover methods to handle failures on service functions. One of the easiest ways is to prepare backup links between ingress and egress VRFs. If a middlebox that is responsible for a service function fails and stops forwarding packets, OSPF processes on the service function network detect the failure by link down or expiring hello timer, and they change next-hops to the backup link that has a higher cost. Another way is to utilize middlebox-specific recovery mechanisms. Recent middleboxes usually have clustering features for failure recovery. Such features can also be applied to service function networks easily because service function networks are traditional IP networks. 
Router-level Failover: Even if a router accommodating service functions fails, service chains should keep forwarding packets from end to end. For this purpose, we slightly modify the unidirectional point-to-point links described in Section 2.2. We describe this modification using the link between To-R2 and From-R1 VRFs in Figure 4 as an example. First, both VRFs import the default route and the user prefixes from the default routing tables and the user VRFs. Second, From-R1 VRF redistributes the default route and the user prefixes as OSPF Type 2 external routes with metric zero to To-R2 VRF. OSPF Type 2 external routes do not add link costs when calculating routes, so that all the next-hops on To-R2 VRF turn toward From-R1 VRF ${ }^{1}$. In this setup, packets redirected to To-R2 VRF also go to From-R1 VRF on Router 2. However, when Router 2 is down, the OSPF neighbor on To-R2 VRF goes down, and To-R2 VRF starts to forward the redirected packets to the external or user networks by the leaked routes. As a result, traffic can keep flowing by OSPF when the routers fail. This failover is also the same in the other inter-router link.

\subsection{Implementation}

Flowchain achieves service chaining with standardized and widely implemented IP routing features: BGP Flowspec, OSPF, and VRF. Therefore, Flowchain-based service chaining infrastructures can be constructed from commercial off-the-shelf IP routers and statically configuring all the features. Even to manage service chains, a route reflector establishing BGP peers with all routers can distribute required Flowspec routes that are statically configured by command line interfaces.

On the other hand, it is also useful to prepare a gateway that translates human- or machine-readable service chain information to BGP update messages. Such a gateway would improve the flexibility of service chain insertion and deletion. Therefore, we implemented a BGP Flowspec speaker for Flowchain. It receives service chain information that contains a target prefix and service functions via the RESTful API and announces proper BGP update messages to the routers. This implementation using ExaBGP [3] is just about 1000 lines of code. The source code of the implementation is available ${ }^{2}$.

\section{DEPLOYMENT CONSIDERATION}

Flowchain-based service chaining is composed of one or more routers, and traffic to which service functions apply needs to pass through the routers. There are three possible ways to steer traffic from user networks to the routers before the external networks.

\footnotetext{
${ }^{1}$ The OSPF Type 2 external with metric zero is not the only method for this failover. The redistributed routes only have to be chosen against the leaked routes on the calculating process in To-R2 VRF.

${ }^{2}$ https://github.com/upa/flowchain
}

A simple way to take traffic to service chaining is for the routers, specifically the user VRFs of the routers, to announce default routes to the Interior Gateway Protocol (IGP) domain. The traffic from user networks then goes to any one of the routers. In this case, the default routing tables of the routers must connect with the default free zone, and they must redistribute the user prefixes to the default free zone to take packets from the external to the user networks through service chains. Another way is to use MPLS. VRF was initially designed for maintaining per-site forwarding tables in the MPLS/VPN paradigm [17]. Thus, utilizing MPLS to bring target traffic to target VRFs is a natural design. Instead of MPLS, some tunneling protocols can be replacements. For example, Generic Routing Encapsulation is widely implemented in current IP router products, and Virtual eXtensible LAN has become popular. Unlike MPLS, such tunneling protocols can adapt to IP-based backbone networks.

Each of these ways has its merits and demerits. Originating default routes do not need additional protocols like MPLS and tunneling; however, the routers comprising service chaining must be placed on the border between the default free zone and the IGP domain. This limitation may restrict backbone designs. On the other hand, MPLS and tunneling approaches can deploy the routers anywhere, for example, in data centers. This offers flexibility to network designs but introduces operation costs in addition to basic IP backbone networks. In other words, this is a design choice for network operators who intend to adopt Flowchain.

\section{EXPERIMENT}

In this section, we demonstrate the feasibility of Flowchain through four experiments. These experiments mainly focus on control aspects of Flowchain-based service chaining because the data plane is commercial off-the-shelf IP routers with the capacity for forwarding packets at wire rate. The first experiment demonstrates the behavior of Flowchain through service function insertion and deletion. The second experiment evaluates the agility of dynamic service chain insertion. The third experiment demonstrates the failover by using OSPF. The last experiment shows the increase of latency due to the length of service chains.

For the experiments, we prepared a simple testbed comprised of two Juniper Networks MX5 routers, a Juniper Networks EX4300 switch, and a DELL R320 server. Figure 5 shows the physical and logical topologies of this testbed. All devices directly connected to the switch by 1 Gbps links. In the logical topology, the server performed as both a user host (ns-usr) and an external host (ns-ext) by separating its network instances using the Linux network namespace. The two MX5 routers, ns-usr, and ns-ext formed an IGP domain using OSPF, and the user VRFs in the MX5 routers announced 


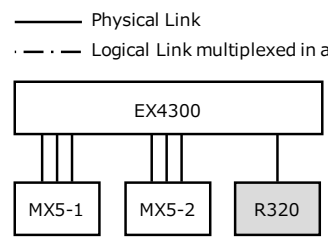

Physical Topology

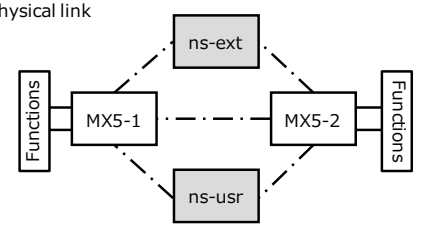

Logical Topology
Figure 5: The testbed topology.

the default routes to ns-usr. The links on this IGP domain were logical links multiplexed in physical links by VLAN. Both MX5 routers used the remaining two physical links to connect with service functions. In this testbed, a service function was just a VLAN through the switch to simplify the experiments. Each VLAN connected to two VRFs in an MX5 router, and it performed as an individual IP network where OSPF ran. In addition, each VRF pair had another VLAN as a backup link. The BGP Flowspec speaker implementation described in Section 2.4 ran on another network namespace and established BGP peers with the MX5 routers.

\subsection{Demonstrating Flowchain}

The first experiment demonstrates the behavior of Flowchain. We sent 100 Mbps TCP traffic from ns-usr to ns-ext through a service chain using iperf3, and measured throughput on three service functions while adding and removing the service functions intermittently. Figure 6 shows the time-line of this experiment. The three lines in Figure 6 indicate throughput of traffic measured at each service function. Add and Remove indicate the times at which we added or removed the service functions from the service chain by the BGP Flowspec speaker. To measure the throughput, we used the Junos Telemetry Interface [14]. Note that the minimum telemetry report interval in current MX routers is 2 seconds; thus, the time resolution of Figure 6 is 2 seconds.

As Figure 6 indicates, Flowchain successfully steered target traffic to target service functions. At 40 seconds, the test traffic flowed through all the three service functions. On the other hand, there were response time lags of a few seconds between when the BGP Flowspec speaker added or removed service functions and when the traffic was actually steered. This is because the routers need time to calculate and install Flowspec routes into their packet forwarding engines after they receive $\mathrm{BGP}$ update messages.

\subsection{Install Time}

Next, we evaluated the response time lags that the previous experiment showed. This time lag affects the agility of dynamic service chain manipulation. In this experiment,

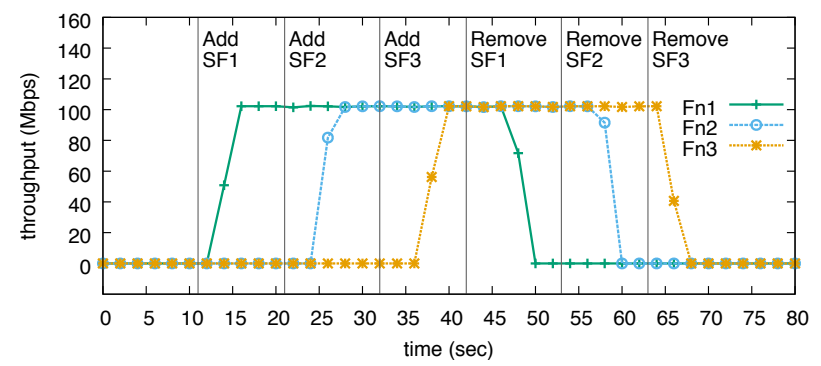

Figure 6: Demonstration of Flowchain.

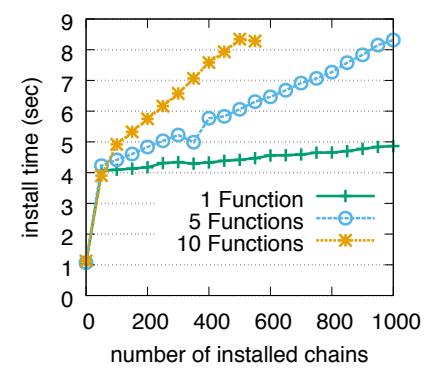

(a) Install time versus the number of installed service chains.

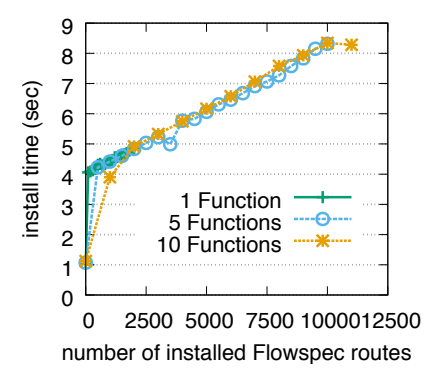

(b) Install time versus the number of installed Flowspec routes.

\section{Figure 7: Time required to install a new service chain.}

we measured the time between when the BGP Flowspec speaker added a service chain and when packets started to flow through the added service chain, which thus represents the time required to install Flowspec routes. To measure this time lag, we sent probe packets from ns-usr to ns-ext every 10 milliseconds. We took the change of the Time-to-Live value of the probe packets as the time at which the service chain was installed into the packet forwarding engine.

Figure 7a shows the result of this experiment. The $\mathrm{x}$-axis indicates the number of installed service chains before the probed service chain was installed. As shown, when there are no installed service chains, the install time is approximately 1 second. After that, the time required to install the service chain linearly increases from about 4 seconds. When all service chains involve one service function, the install time reaches 5 seconds with 10000 chains. On the other hand, the time required to install increases with the number of service functions involved in the service chains. It exceeds 8 seconds with over 950 service chains involving five functions, or over 500 service chains involving 10 functions.

The time required to install a service chain depends on the number of installed Flowspec routes: the increase of Flowspec routes increases the time lag. Figure $7 \mathrm{~b}$ shows the same experiment result from the viewpoint of the number of Flowspec routes. We see that the time required to install 
Practical Service Chaining based on IP Routing

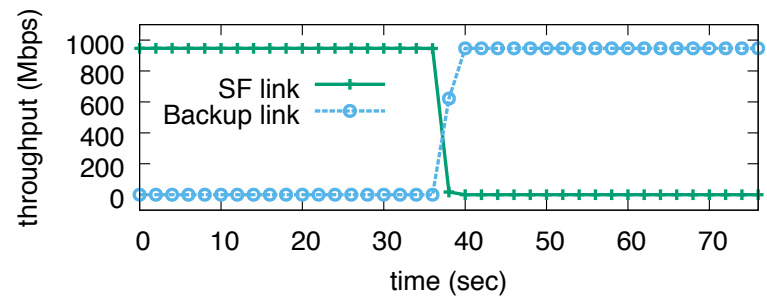

Figure 8: Service function-level failover.

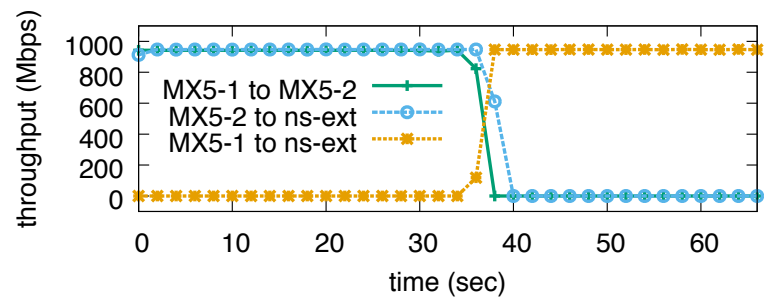

Figure 9: Router-level failover.

Flowspec routes increases linearly with the number of installed Flowspec routes. However, the rate of increase is approximately 1 second per 2500 Flowspec routes. Although the time required to install Flowspec routes depends on the scale of service chains, we conclude that Flowchain can dynamically add and remove service chains within a practical time, that is, several tens of seconds.

\subsection{Failover}

The third experiment demonstrates fault tolerance in Flowchain. Figure 8 shows service function-level failover on a service chain through one service function on MX5-1. In this experiment, ns-usr sent 1 Gbps TCP traffic to ns-ext through the service function, and we then disabled the service function. It shows the transition of throughput on the link of the service function and the associated backup link. After disabling the link of the service function, OSPF running on the service function network detected link down and recovered by selecting the backup link within 4 seconds $^{3}$.

Figure 9 demonstrates router-level failover. In this scenario, a service chain passed through a service function on MX5-1 and a service function on MX5-2. Before emulating the failure on MX5-2, traffic reached ns-ext through the MX52 to ns-ext link. When we disabled the logical link between MX5-1 and MX5-2, the OSPF process on MX5-1 detected it and detoured the traffic to ns-ext directly by routing table changes described in Section 2.3. As a result, throughput on the MX5-1 to MX5-2 link and the MX5-2 to ns-ext link decreased, and MX5-1 started to forward packets to ns-ext. In this way, Flowchain achieves fault tolerance by OSPF.

\footnotetext{
${ }^{3}$ The time resolution in this experiment was also 2 seconds.
}

AINTEC '18, November 12-14, 2018, Bangkok, Thailand

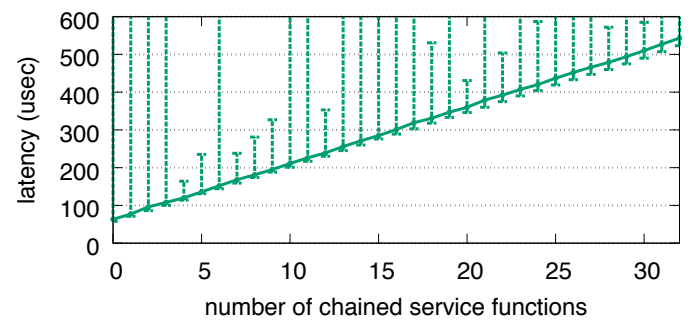

Figure 10: Latency versus the number of chained service functions.

\subsection{Latency versus Service Chain Length}

So far, we have demonstrated that Flowchain works from the control perspective. The fourth experiment therefore measured the effect of service chaining from the packet forwarding perspective. It is naturally expected that taking packets through multiple service functions increases latency. We measured this increase on the testbed. In this experiment, we sent probe packets in which timestamps are embedded from ns-usr to ns-ext, and measured the one-way latency through a service chain. Note that the timestamp was microsecond scale, and ns-usr and ns-ext are separated namespaces in the same host; therefore, there is no time clock difference.

Figure 10 shows the result of this experiment. The $\mathrm{x}$-axis indicates the number of chained service functions, and the $y$-axis indicates the latency from ns-usr to ns-ext. Note that the result is median, minimum, and maximum of 1000 probe packets on each number of chained service functions. This result confirms that the latency through the service chain increases with the number of chained service functions. The latency for one service function is $10-20$ microseconds. In this testbed, a service function is just a VLAN through the EX4300. Thus, the latency for a service function is composed of the redirection by a Flowspec route, transmission to the wire, switching, reception from the wire, and redirection by a Flowspec route again. All of these steps are performed on the hardware of the router and the switch. In sum, this result demonstrates the baseline latency due to chaining, and it was found to be minimal. In actual deployment, middleboxes would add latencies to this baseline latency.

\section{DISCUSSION}

Flowchain demonstrates that practical service chaining can be achieved by redirection using BGP Flowspec, OSPF, and VRF. At the same time, its packet steering still relies on traditional IP routing and forwarding. This section briefly discusses its potential limitations compared with SDN-based approaches and other packet forwarding mechanisms.

SDN-based Approaches: Although SDN-based service chaining requires additional development costs and introduces complexity into network operation, it can achieve what 
IP cannot, for example, fine-grained traffic control. Because IP routing is hop-by-hop, shortest-path routing, it cannot handle traffic at the connection level. OpenNF [7] achieved tight service level agreements, highly accurate traffic monitoring, and manipulation by connection state-based packet handling. In Flowchain, the resolution of traffic control (e.g., traffic engineering and assurance of quality of services) is limited to the capability of traditional IP routing.

BGP VPN: BGP VPN-based service chaining has been proposed and discussed [5]. We are inspired by this method-it also puts service functions between ingress and egress VRFs. In contrast to Flowchain, which achieves service chains by Flowspec and the pre-configured topologies, BGP VPN-based service chaining proposes a new route target, called servicetopology-RT, to describe service chains. If this enhancement completes the standardization process and production routers support it, IP routing-based service chaining would achieve more flexible topology designs than Flowchain.

Segment Routing: Segment Routing (SR) is a new packet forwarding mechanism that achieves paths by the source routing paradigm. Utilizing SR for service chaining has also been discussed [2]. For controlling SR-based networks, Path Computation Element Protocol (PCEP) deploys paths [20]. In other words, SR is a data plane, and PCEP is its southbound API. Therefore, to achieve service chaining with SR, the programming and development of controllers are required. However, unlike SDN, this mechanism does not introduce additional complexity due to dedicated mechanisms into the data plane, because SR is a standardized and fixed packet forwarding mechanism.

\section{CONCLUSION}

In this paper, we have demonstrated that practical service chaining can be achieved by leveraging current IP routing techniques. Flowchain utilizes the redirection of BGP Flowspec instead of implementing paths in packet forwarding mechanisms, and OSPF for routing and failover. Unlike SDN-based service chaining, it does not require additional development costs for both data and control planes, and it allows the application of well-matured techniques and the knowledge of traditional IP routing. The feasibility of Flowchain was demonstrated by our experiments. It was shown that Flowchain can dynamically change service chains in a few seconds, and failover using OSPF achieves practical fault tolerance. Moreover, the baseline latency due to service chaining is minimal. We believe that Flowchain would contribute to practical service chaining as well as future discussion and comparison for service chaining methods.

\section{ACKNOWLEDGMENT}

This work was originally designed for Interop Tokyo 2018 ShowNet. The authors would like to thank all Interop Tokyo 2018 ShowNet NOC team and contributor members.

\section{REFERENCES}

[1] Fred Baker, David L. Black, Dr. Kathleen M. Nichols, and Steven L. Blake. 1998. Definition of the Differentiated Services Field (DS Field) in the IPv4 and IPv6 Headers. RFC 2474. (Dec. 1998).

[2] Francois Clad et al. 2018. Service Programming with Segment Routing. Internet-Draft draft-xuclad-spring-sr-service-programming-00. Internet Engineering Task Force. Work in Progress.

[3] Exa-Networks. 2018. The BGP swiss army knife of networking. https: //github.com/Exa-Networks/exabgp. (Jul 2018).

[4] Seyed Kaveh Fayazbakhsh et al. 2014. Enforcing Network-Wide Policies in the Presence of Dynamic Middlebox Actions using FlowTags. In 11th USENIX Symposium on Networked Systems Design and Implementation (NSDI 14). USENIX Association, Seattle, WA, 543-546.

[5] Rex Fernando et al. 2018. Service Chaining using Virtual Networks with $B G P$ VPNs. Internet-Draft draft-ietf-bess-service-chaining-04. Internet Engineering Task Force. Work in Progress.

[6] Clarence Filsfils et al. 2018. Segment Routing Architecture. RFC 8402. (July 2018).

[7] Aaron Gember-Jacobson et al. 2014. OpenNF: Enabling Innovation in Network Function Control. In Proceedings of the 2014 ACM Conference on SIGCOMM (SIGCOMM '14). ACM, New York, NY, USA, 163-174.

[8] Joel M. Halpern and Carlos Pignataro. 2015. Service Function Chaining (SFC) Architecture. RFC 7665. (Oct. 2015).

[9] W. John et al. 2013. Research Directions in Network Service Chaining. In 2013 IEEE SDN for Future Networks and Services (SDN4FNS). 1-7.

[10] Christoph Loibl. 2017. BGP Flow Specification Interoperability Lab. https://ripe74.ripe.net/archives/video/167/. (May 2017).

[11] Pedro R. Marques, Jared Mauch, Nischal Sheth, Barry Greene, Robert Raszuk, and Danny R. McPherson. 2009. Dissemination of Flow Specification Rules. RFC 5575. (Aug. 2009).

[12] A. M. Medhat, T. Taleb, A. Elmangoush, G. A. Carella, S. Covaci, and T. Magedanz. 2017. Service Function Chaining in Next Generation Networks: State of the Art and Research Challenges. IEEE Communications Magazine 55, 2 (February 2017), 216-223.

[13] A. A. Mohammed et al. 2016. SDN controller for network-aware adaptive orchestration in dynamic service chaining. In 2016 IEEE NetSoft Conference and Workshops (NetSoft). 126-130.

[14] Juniper Networks. 2018. Overview of the Junos Telemetry Interface. https://www.juniper.net/documentation/en_US/junos/topics/ concept/junos-telemetry-interface-oveview.html. (Jun 2018).

[15] Zafar Ayyub Qazi et al. 2013. SIMPLE-fying Middlebox Policy Enforcement Using SDN. In Proceedings of the ACM SIGCOMM 2013 Conference on SIGCOMM (SIGCOMM '13). ACM, New York, NY, USA, 27-38.

[16] Paul Quinn, Uri Elzur, and Carlos Pignataro. 2018. Network Service Header (NSH). RFC 8300. (Jan. 2018).

[17] Yakov Rekhter and Eric C. Rosen. 2006. BGP/MPLS IP Virtual Private Networks (VPNs). RFC 4364. (Feb. 2006).

[18] Elisa Rojas et al. 2018. Are We Ready to Drive Software-Defined Networks? A Comprehensive Survey on Management Tools and Techniques. ACM Comput. Surv. 51, 2, Article 27 (Feb. 2018), 35 pages.

[19] Colin Scott et al. 2014. Troubleshooting Blackbox SDN Control Software with Minimal Causal Sequences. In Proceedings of the 2014 ACM Conference on SIGCOMM (SIGCOMM '14). ACM, New York, NY, USA.

[20] Siva Sivabalan et al. 2018. PCEP Extensions for Segment Routing. Internet-Draft draft-ietf-pce-segment-routing-12. Internet Engineering Task Force. Work in Progress.

[21] Ying Zhang et al. 2013. StEERING: A software-defined networking for inline service chaining. In 2013 21st IEEE International Conference on Network Protocols (ICNP). 1-10. 